\title{
DATOS, INFORMACIÓN Y EVALUACIONES URBANAS INTEGRADAS PARA LA IMPLEMENTACIÓN Y SEGUIMIENTO DE LAS POLÍTICAS EUROPEAS PARA CIUDADES SOSTENIBLES
}

\author{
D. Christoph Schröder \\ Msc. en Geografía de la Universidad de Bonn. \\ Especialista en análisis medioambiental y GIS. European Topic Centre
}

Artículo Recibido: 21/01/2015

Artículo Aceptado: 03/02/2015

\section{Políticas Europeas sobres sostenibilidad urbana}

Las ciudades van cogiendo cada vez más protagonismo en las políticas, estrategias y legislación europeas al representar tanto el origen de muchos problemas ambientales (y sociales) como un laboratorio de soluciones para conseguir lugares sostenibles y prósperos. Su importancia demográfica (el $75 \%$ de los europeos viven en las ciudades y se prevé que en el año 2030 se estabilizará en alrededor del $80 \%$ ) y sus cifras de consumo de recursos y de la contaminación que ejercen, las convierte en un objeto de políticas y estrategias de primer orden.

A nivel Europeo, esta tendencia se ha manifestado por varias comunicaciones entre las que destaca la "Comunicación de la Comisión al Consejo y al Parlamento Europeo sobre una Estrategia temática para el medio ambiente urbano" (COM(2005) 718$)^{41}$ que subraya que en las ciudades se concentran muchos problemas medioambientales, pero también que son el motor económico y el centro de operaciones de los negocios y la inversión. La calidad de vida de la población urbana depende directamente del estado del entorno urbano, y tiene vertientes tanto física como psicológica, estrechamente interrelacionadas. La calidad de vida que entendemos como la expresión subjetiva de la sostenibilidad urbana, se basa en la exposición a y la percepción de factores ambientales y socio-económicos que se pueden y deben medir con el fin de apoyar la toma de decisiones adecuadas.

Esta reflexión se profundiza en el Programa General de Medio Ambiente de la Unión hasta 2020 «Vivir bien, respetando los límites de nuestro planeta» (COM(2012) 710) ${ }^{42}$. Entre los nueve objetivos se encuentra el de aumentar la sostenibilidad de las ciudades de la Unión. El Programa manifiesta que la mayoría de las ciudades se enfrentan a toda una serie de problemas ecológicos comunes, en particular una calidad del aire deficiente, niveles de ruido elevados, emisiones de GEI, escasez de agua, inundaciones y tormentas, lugares contaminados, zonas industriales abandonadas y residuos. Por otra parte, las ciudades de la UE marcan la pauta en sostenibilidad urbana y son, con frecuencia, pioneras en la aplicación de soluciones innovadoras a problemas ambientales. Son cada vez más las ciudades europeas que

\footnotetext{
${ }^{41} \mathrm{http}: / /$ ec.europa.eu/environment/urban/pdf/com_2005 0718 es.pdf

$42 \mathrm{http}: / /$ ec.europa.eu/environment/newprg/
} 


\section{WPS Review International on Sustainable Housing and Urban Renewal (RI-SHUR)}

sitúan la sostenibilidad ambiental en el centro de sus estrategias de desarrollo urbano. Para aumentar la sostenibilidad de las ciudades de la UE, el Programa pretende garantizar que, para 2020 la mayoría de las ciudades de la UE estén aplicando políticas de ordenación y diseño sostenibles del espacio urbano. A tal fin, sería necesario, en particular:

a) determinar y acordar un conjunto de criterios para evaluar el comportamiento ambiental de las ciudades, teniendo en cuenta los impactos económicos y sociales;

b) garantizar que las ciudades dispongan de información sobre la financiación de medidas para mejorar la sostenibilidad urbana, y que tengan acceso a esos fondos

El conjunto de criterios para evaluar el comportamiento ambiental de las ciudades puede directamente traducirse en un sistema de indicadores y umbrales relacionados. Para poder definir, crear y comparar los indicadores al igual que para el monitoreo y el seguimiento de las políticas urbanas, hacen falta datos e información específicos.

Como muestra la figura 1, hay una gran diversidad de datos que se puede utilizar para la evaluación de áreas urbanas a escala europea. Estos datos difieren en el grado de resolución espacial y la cobertura.

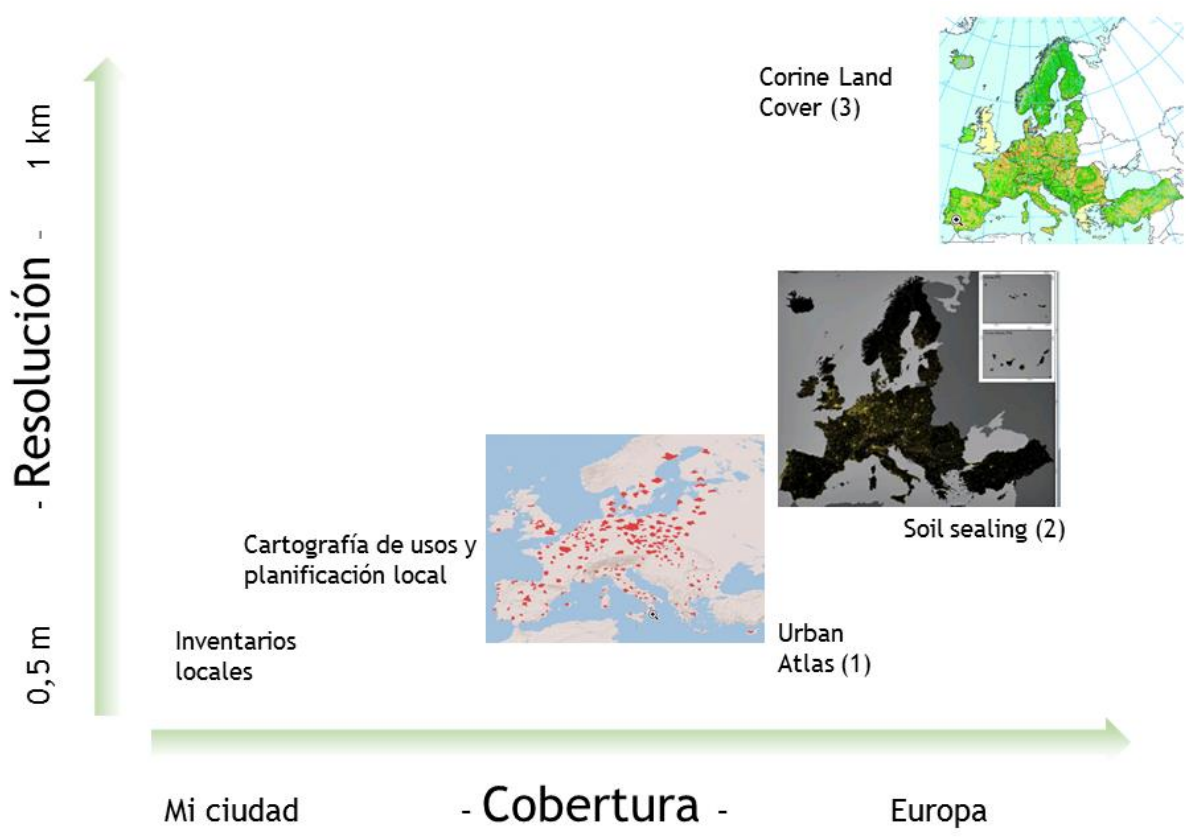

\section{Análisis urbano a nivel Europeo: La Agencia Europea de Medio Ambiente y Centro Temático Europeo de Suelo y Sistemas Terrestres y Urbanos}

De hecho, la Agencia Europea de Medio Ambiente (AEMA), apoyada por el Centro Temático Europeo sobre Suelo y Sistemas Terrestres y Urbanos (ETC-ULS, en sus siglas ingleses) llevan años trabajando en la evaluación de áreas urbanas a escala europea con el objetivo de apoyar y monitorear las políticas Europeas sobre medio 


\section{WPS Review International on Sustainable Housing and Urban Renewal (RI-SHUR)}

ambiente urbano. Los sistemas urbanos son uno de las áreas estratégicas de la AEMA en las que informan de la implementación de las políticas europeas. En esta área se trabajan distintos enfoques desde el crecimiento difuso en las zonas peri-urbanas hasta el análisis de los servicios que prestan los ecosistemas urbanos, pasando por la delimitación de zonas urbanas, la calidad de aire, el análisis del cambio de uso y su impacto en relación con la adaptación al cambio climático. Todos estos informes y análisis se basan en los datos disponibles y accesibles con cobertura europea puesto que el objetivo final de las evaluaciones es la comparación entre ciudades y el apoyo a los responsables de toma de decisiones locales en cuanto a situar su ciudad y sus características en el contexto europeo.

En esta revisión se hace hincapié en dos temas de relevancia que además cuentan con una base de datos muy amplia.

El crecimiento urbano es un proceso muy actual y dinámico que afecta a muchas partes del continente europeo. Alrededor de tres veces el tamaño de Luxemburgo ha sido ocupado por zonas urbanas en el periodo 2000-2006 (EEA 2006), ocupando zonas agrícolas, forestales, de pasto o de vegetación natural. Además, el sellado de suelo, relacionado con la urbanización, provoca una reducción de las funciones de suelos (producción de biomasa, secuestro de carbono, etc.). Actualmente, se está trabajando en la mejora de los indicadores y la modelación del crecimiento urbano para poder relacionar variables económicos y sociales con la ocupación física del espacio (Jaeger \& Schwick 2014).

Por otro lado, se está trabajando en la mejora de los indicadores sobre la sostenibilidad en ciudades europeas para elaborar una tipología de ciudades. Un paso previo para la mejora de indicadores es la colección de datos comparables y de máxima cobertura. EI ETC-ULS ha conseguido recopilar una amplia cantidad de datos de diferentes fuentes (Eurostat, AEMA, estadísticas sectoriales) permitiendo elaborar los indicadores propuestos para un amplio número de ciudades (aprox. 400 ciudades europeos). Estos indicadores cubren diferentes dominios temáticos: Socio-economía, calidad de aire, morfología física, uso de recursos (agua, energía, suelo), gobernanza. Así, se permite avanzar hacia una visión completa de la sostenibilidad de las áreas

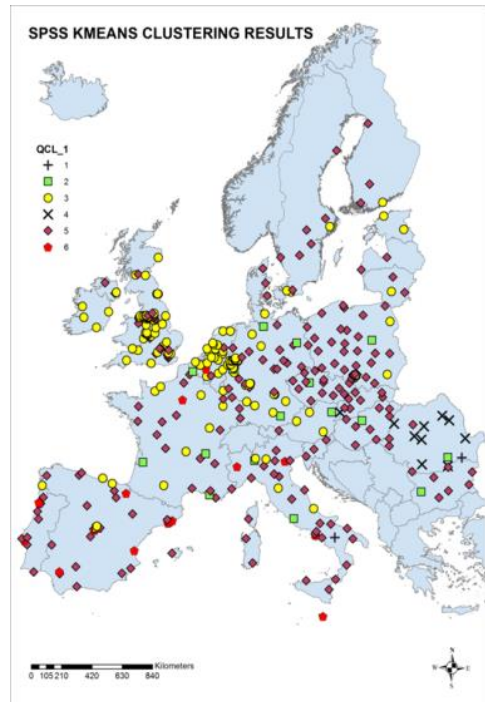
urbanas y poder medirlas con datos comparables y consistentes.

A partir de esta base de datos y la definición de indicadores, el ETC-ULS ha avanzado en crear una tipología de ciudades para Europa que facilita una visión comparativa de los tipos más característicos de ciudades. Actualmente, se está trabajando en dicha tipología que se basa en un análisis geo-estadístico (análisis clúster) que agrupa ciudades con características similares en grupos que, a continuación pueden ser monitorizados en conjunto (ver figura 2).

La gran ventaja de esta metodología es su potencial para ser trasladada a otras escalas. De esta forma, se 


\section{WPS Review International on Sustainable Housing and Urban Renewal (RI-SHUR)}

podrán elaborar tipologías de barrios o distritos en ciudades que dispongan de datos suficientes. Además, será posible integrar otros datos relevantes a nivel local como son los datos sobre viviendas y rehabilitación.

Dichas tipologías permitirán a los responsables de la planificación y ordenación urbana de tener una visión integrada de la sostenibilidad de la ciudad y actuar conforme a ello para la mejora de la misma.

\section{Redes y colaboración}

El trabajo que está realizando el ETC-ULS es un trabajo colaborativo y en red. Para ello, la Agencia Europea de Medio Ambiente coordina y organiza la iniciativa IUME (towards an Integrated Urban Monitoring in Europe ${ }^{43}$ ) bajo la supervisión de la Dirección General de Medio Ambiente de la Comisión Europea. Como grupo de trabajo colaborativo, se basa en la contribución voluntaria e informal entre distintas instituciones europeas que tratan, desde distintos ángulos, los procesos urbanos y su impacto ambiental. Participan, aparte de DG Env y la EEA, el ETC SIA, DG Regio, Eurostat, los Joint Research Centre (JRC) de la Comisión Europea, las redes de ciudades ICLEI y Eurocities, ESPON, OECD y varias agencias nacionales.

Puesto que hay varias iniciativas de monitoreo urbano llevándose a cabo en Europa careciendo un planteamiento integrado, el objetivo principal de esta plataforma es el intercambio de información entre los participantes y el desarrollo del concepto de monitoreo integrado. De esta forma, la eficiencia de trabajo puede aumentarse, mientras lagunas de datos e información pueden taparse gracias a la interacción de los distintos actores involucrados.

Las distintas instituciones que trabajan en el monitoreo y el análisis del desarrollo urbano en Europa, organizan regularmente (cada seis meses) jornadas que ofrecen el ambiente idóneo para compartir información sobre las investigaciones y desarrollos más recientes y para acordar trabajo futuros, o bien individuales o conjuntos. Al mismo tiempo y gracias a la participación de técnicos de varias Direcciones Generales de la Comisión Europea, los participantes están al tanto del desarrollo y de la demanda política tanto a nivel europeo como nacional, así como de las convocatorias más relevantes en la temática urbana.

\section{Conclusiones}

Hay un acuerdo generalizado que el monitoreo y análisis de la sostenibilidad de las áreas urbanas es imprescindible para poder elaborar acciones focalizadas y eficaces. El análisis de la situación actual y de las tendencias es fundamental para la definición de políticas europeas. En cambio, estas políticas requieren posteriormente un seguimiento estructurado y amplio para poder identificar los éxitos y debilidades de las mismas.

Para todo ello, es necesario disponer de datos para poder elaborar indicadores que nos den información sobre el grado de cumplimiento de las políticas. Estos datos actualmente son todavía dispersos y no completos, por lo que hace falta un esfuerzo

\footnotetext{
${ }^{43}$ http://iume.ew.eea.europa.eu/ 


\section{WPS Review International on Sustainable \\ Housing and Urban Renewal (RI-SHUR)}

hacer accesible y comparable la gran cantidad de datos que albergan las administraciones a distintos niveles.

Una vez disponible los datos, tocará a los expertos integrarlos para obtener la información requerida y poder apoyar sustancialmente en la definición y el seguimiento de las políticas europeas y nacionales. Ya se han alcanzado hitos muy importantes en esta dirección, pero todavía queda mucho camino por recorrer para obtener una base de información suficiente para satisfacer las necesidades de las políticas y de gestión acerca de la sostenibilidad urbana.

\section{Bibliografía}

EEA (2006): Urban Sprawl in Europe. The ignored challenge. EEA Report 10/2006.

JAEGER, J. A.G. \& SCHWICK, C. (2014): Improving the measurement of urban sprawl: Weighted Urban Profileration (WUP) and its application to Switzerland. Ecological Indicators 38: 294-308. 
WPS Review International on Sustainable

Housing and Urban Renewal (RI-SHUR)

\section{PROPUESTAS DE ACTUACIÓN}

